\title{
A Practical Axial Compressor Design Optimization Approach Based on Gas Turbine Operation
}

\author{
Mohammad Reza Pakatchian ${ }^{1}$, Hossein Saeidi ${ }^{2}$ \\ ${ }^{1}$ MAPNA Turbine Engineering and Manufacturing Company (TUGA) \\ MAPNA blv. Fardis, Karaj- I.R. Iran \\ pakatchian.mohammad@mapnaturbine.com; saeidi.hossein@mapnaturbine.com
}

\begin{abstract}
In the current study, it is focused on blade optimization of compressor to achieve improved performance characteristics. Due to dependency of mass flow rate on the inlet temperature of the gas turbine, temperature changes influence on compressor performance and efficiency. In order to enhance the working conditions at design and off-design operation, an automated design process is applied. The process has three main steps including parametrization of the geometry, numerical simulation of flow and optimization design approach. Stochastic design approach is utilized for optimization. The objective of this improvement will push the airfoil geometry in a way that minimum loss value, extended acceptable off-design operation in constant exit flow angle can be achieved with being focused on hot day's operation. The considered case in the present study is a compressor of MGT-70 heavy-duty gas turbine and the optimization focuses on the first four stages. Based on numerical simulation of optimized compressor, $1 \%$ enhancement in efficiency in all operating conditions is achievable. Moreover, the mass flow rate can be enhanced roughly up to $0.8 \%$ and $1 \%$ for design and off-design conditions, respectively. After assembling the new developed parts, the first upgraded prototype of the gas turbine has been tested in sixth Unit of Parand power station. More than 600 signals of pressure and temperature in circumferential and radial directions were extracted from compressor section. The results show good agreement predicted in range inlet flow angle between measurements and theoretical targets.
\end{abstract}

Keywords: Axial compressor, Shape optimization, Gas turbine operation, Geometry parametrization.

\section{Introduction}

Due to increase in demand for electricity and environmental issues related to electricity generation, it is necessary to enhance the efficiency of electricity producing. The share of gas turbines in electricity generation is noticeable; therefore, improving their performance will result in more proper energy systems. Performance of gas turbines depends on the ambient conditions. Seasonal climate changes from cool to hot days with conjunction to increasingly power demand will obligate the designer to design in a condition with need to have stable performance operation in an acceptable operating efficiency.

Variations in ambient condition effect on mass flow rate and efficiency of compressors, as a consequence, the overall performance of the gas turbines will be influenced. In order to enhance the gas turbine characteristics, it is necessary to optimize each gas turbine components, especially its compressor, by considering operating conditions.

There are several studies on optimizing compressor blades [1]-[5]. The optimization approaches are commonly categorized as inverse and direct design processes. In inverse design optimization, on the basis of inlet flow angle and thermodynamic conditions, the best distribution of velocity or pressure around the airfoil are prescribed. Iterative process is employed to obtain the target geometry.

In this method, by using parametrized geometry and stochastic algorithm, the optimized velocity or pressure distribution will match to the desired pattern. This approach requires designer's knowledge and experience, since the velocity distribution must be defined so that different aspects of inlet/outlet flow angles and boundary layer conditions could be considered at the related inlet Mach number. Disability to off-design optimization is one of disadvantages of this method [1].

More common optimization tools are on the basis of direct design approach [2]-[4]. In direct design method, secondary flow properties such as profile loss of airfoil are considered with constant input geometry, inlet and outlet flow angles. Sanger [5] investigated subsonic airfoil and initiated an automated numerical optimization process on it. Gradient-base approaches and stochastic algorithms are among the most conventional methods, which are applicable for direct design optimization. In the gradient-base methods, derivatives of all objectives and constraints are employed to define the optimization search direction [6]. Stochastic design optimizations work on the basis of evaluation strategies and genetic algorithm. Using these 
methods can lead to appropriate results in the cases of noisy domains which have multi objective function and complex geometries.

In the present study, the base load performance of MGT-70 gas turbine is investigated in various ambient temperatures. Afterwards, optimization algorithm is represented based on aerodynamic specifications. The defined multi objective function for the optimization is improving both design and off-design operations. Finally, the results are analyzed and compared with experimental data.

\section{Numerical Simulation of Compressor}

Computational Fluid Dynamic (CFD) is broadly applied to numerically analyze turbomachines. Several approaches are represented to model multi stage turbomachines such as axial compressors and turbines. In the first step of the current research, axial flow compressor of MGT-70 is investigated at the design point. The compressor has 16 stages and an inlet guide vane (IGV). The bleed valve exists in the fifth stage between rotor and stator. Numerical simulation is carried out at the base load for a defined static backpressure.

ANSYS CFX-16 is applied to simulate the compressor. Turbo Grid 16.0 is used to generate structured mesh. Structured (mapped) hex meshes is used since the passages of the blade do not have complex geometry and can be considered as a "clean CAD" model. In order to connect the mesh regions, which are not matched, General Grid Interface (GGI) is applied as interface strategy [8], [9].

In the first step of this study, a 3D simulation is carried out on the compressor at the design point working condition. Static backpressure of the compressor is the only parameter that can be utilized to change for obtaining the best pressure ratio matching.

In the next step, the results of the total machine performance are extracted. Comparison between simulation results and the data obtained during its operation in some power plants indicates the accuracy of performed simulation. Appropriate agreement founded between predictions and experimental results, both in the details of the flow field and the integral prediction of operating range in the compressor. In Fig. 1, actual data and simulation results are compared. In this figure $\mathrm{PR}^{*}$ is normalized pressure ratio, $\mathrm{M}^{*}$ is normalized mass flow.

Based on thermodynamic simulation and available data of the plants, temperature variations can cause degradation in performance of the compressor. Due to this fact, some attempts are done to achieve an acceptable operation by providing flattened performance operation [10]-[14]. In the current research, numerical simulation of compressor at offdesign working condition is evaluated at the base load in four different ambient temperatures including $0,15,35$ and 45 degree of Celsius. The obtained results are represented in Table 1.

Table 1: Non-dimensional results of gas turbine base operation at various ambient temperatures.

\begin{tabular}{|l|c|c|c|c|}
\hline Inlet Temperature (C) & 0 & 15 & 30 & 45 \\
\hline Corrected Speed & 1.027 & 1 & 0.975 & 0.95 \\
\hline Mass flow & 1.048 & 1 & 0.938 & 0.877 \\
\hline Isentropic Efficiency & 0.991 & 1 & 0.998 & 0.996 \\
\hline Pressure Ratio & 1.048 & 1 & 0.94 & 0.868 \\
\hline
\end{tabular}




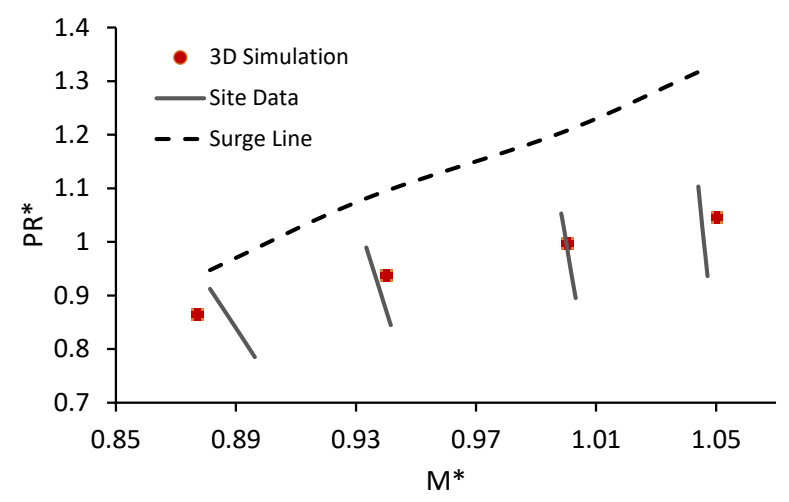

Table 2: Error values for mass flow rate.

\begin{tabular}{|l|l|}
\hline Inlet Temperature (C) & Error (\%) \\
\hline 0 & 0.48 \\
\hline 15 & 0.038 \\
\hline 30 & 0.45 \\
\hline 45 & -0.938 \\
\hline
\end{tabular}

Fig. 1: Compressor characteristics base on simulation and site data.

As shown in Table 1, increase in inlet temperature leads to reduction in both mass flow and efficiency; therefore, the generated power would be decreased. The differences between inlet mass flow in site data and simulations are presented in Table 2.

Roughly, temperature changes in Iran vary from $\left(\sim 45^{\circ} \mathrm{C}\right)$ in hot days to $\left(\sim 0^{\circ} \mathrm{C}\right)$ in cool days; therefore, it is necessary to enhance the performance of the working gas turbines in ambient conditions with high temperatures. Due to this fact, the optimization is concentrated on augmentation of performance at high inlet temperatures. Mach number at the inlet of gas turbines affects the operating range of it, since inlet Mach number of starting stages limits their attainable working range.

The sections which generate blades should be compatible with working conditions. Variation in ambient temperature has impact on operation of blade sections at off-design conditions in various Mach numbers and inlet axial velocities.

In prospective view of velocity triangles, by changing axial velocity, inlet relative flow angle could be varied with change in the axial velocity. This will push the cascade characteristics in a way that maximum loss value or stall condition be predominant. Spanwise Variations of inlet flow angles in the first stage of the compressor are depicted in Fig. 2. In this figure RHD refer to Rotor Hot ambient inlet Design condition and SHD is Stator Hot ambient inlet Design condition. The represented data are plotted according to deviations from the ISO base load operation. These generated angle bounds are obtained by applying numerical simulations in various ambient temperatures ( 0 to 45 degree of Celsius).

Results of the modeling indicate that, at low ambient temperatures incidence flow angle is negative, while it would be positive at high ambient temperatures. Each of these blades is generated on the basis of some 2D sections. In order to achieve more appropriate performance, proper loss value and attainable working condition should be enhanced at the specific inlet Mach values.

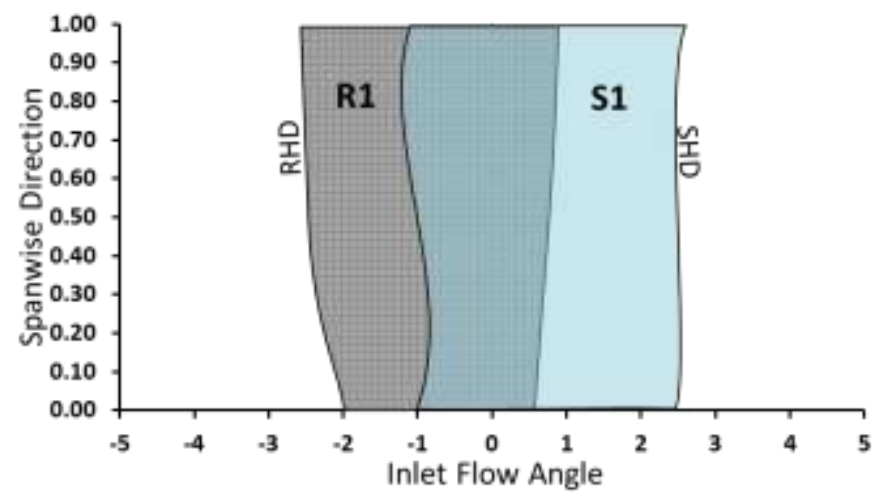

Fig. 2: Effect of ambient temperature changes (from 0 to 45 degree of Celsius) on the first stage inlet flow angle variations.

FFHMT 131-3 


\section{Optimization Process}

The important sections used in this design activity includes adaptive geometry parametrization, an evolutionary optimization algorithm, most popular surrogate model and a 3D high efficient aero-mechanical design software for numerical simulations.

The degree of freedom can be changed for each compressor blade shape based on the complexity of design inlet parameters. The optimization process is developed based on evolutionary algorithms, while the geometrical and aerodynamic properties are considered as constraints.

In order to reduce the computational cost of calculations, Artificial Neural Networks is used as surrogate model along with the numerical simulations. At the beginning, ANN should be trained by appropriate selected number of tests, which are obtained by numerical simulations. This is done by implementation of maximum and minimum values of influential variables by employing Design of Experiments (DOE) method. Regarding to the large number of design variables and time consumption of process, fractional factorial method has been used. The number of variables is another key parameter affecting the degree of freedom; as a consequence, the number of data base sections for the rotor of first stage was defined equal to 400 . The data base was modified during the optimization process enabling achievement of a more accurate design model, this process was done until the ANN was trained. By generation of data base, interactions and accuracy of selected bound for each variable is evaluated.

According to the trained ANN, the results of new geometry are generated, some special estimations of ANN numerically modeled and the target results added to the data base. This scope is done until the results of ANN converged to the numerical results.

\subsection{Geometry Parametrization}

The geometry becomes parameterized with conventional Bezier method by using of an in-house provided tool. Geometry is defined by certain number of parameterized airfoil sections with distribution of camber line and thickness, each 2D section can be parameterized by fifteen Bezier control points, five control points for camber line and ten control points for thickness distribution.

Definition of changes in control points depends on the geometry constraints and the properties of the boundary condition. In this design, some control points in blade leading and trailing edges are locally fixed. The variations in control points of each section depend on the other sections; this can reduces the disturbances occurring on the generated blade surface. The stacking line is parameterized by five control points; this can give access to change the blade geometry near the walls in sweep and lean angles. In Spanwise direction the definition of movable control points has been differed between rotary and stationary blades, this is due to the different design of this machine in rotor and stator blades. According to the, the stator blade is fixed at the tip.

Selection of appropriate control points and its margin of variation from minimum to maximum value are very important to obtain the best geometry with least amount of dissonance. Selecting inappropriate control points with unsuitable margins causes more computational time with some errors on the design procedure.

\subsection{Calculation Procedure}

The generated geometries are evaluated on design and off-design operating points based on the sensed boundary conditions. One of the most important off-design effects during the engine operation is variation of inlet flow angle. This can happen during the part load operation and seasonal atmospheric changes; this means that each blade shape should be in an acceptable operating range [15]. Controlling of geometrical and aerodynamic constraints is on the other side of this optimization process. In order to achieve it, degree of freedom is adopted in control points with taking into account the blade location in rotary and stationary blades.

According to Fig. 3, chord length, rotational speed, number of blades, span height, blade root location and mechanical aspects would limit the freedom of new geometries. With accounting to this specific situation, Bezier control 
points would affect the distributions on chamber line and thickness with stagger and camber angles for the generation of new geometries.

Blade-to-blade (S1) computations are performed using Proven and commercialized software Gambit 2.4.6 and Fluent 6.3 for grid generation and flow CFD simulations, respectively.

Each blade is described by a number of profile sections on the conical surface based on the stream lines position. These profile sections used as 2D sections for cascade analysis and 2D optimization.

Profile sections have been used in grid generator software with specified height and blade number. Boundary layer mesh is used near the airfoil and unstructured mesh has been used far from the walls. In this generation, upper and lower bounds of domain have periodic boundary conditions and there is used total inlet pressure and static outlet pressure as boundary conditions for inlet and outlet region, respectively.

For each blade row, 3D flow simulation has been done before optimization based on the initial blade geometry. Afterwards, according to the selected row, span wise flow properties are extracted as the cascade boundary condition.

Number of individuals in each data base depends on optimal generated airfoils that are extracted in groups with wide range of acceptable design variables near convergence of optimized airfoil. In the three-dimensional view, the blade shape is formed with 2D airfoils which are generated by ANN or stored on the relevant data bases.

An iterative process was employed to achieve Mach number at design and off-design simulations. Simulation started by initializing the outlet static gauge pressure. After each solution, reported inlet Mach number was checked with the reference value. In the cases the difference was more than $0.5 \%$, outlet static gauge pressure was corrected and another simulation was conducted.

\subsection{Fitness function calculation}

In order to achieve optimal geometry based on the geometrical parameters, it is important to properly define the objective function both in 2D and 3D algorithms (Fig. 3). For this activity, the genetic algorithm with weighted sum method was selected for optimization. All the parameters that may positively affect the performance in design and off-design conditions are considered in two formulations defining optimization quality in $2 \mathrm{D}$ and 3D algorithms. In 2D cascade geometries, objective function is defined according to cascade properties where properly retrofit the loss value in choke and stall conditions, increasingly the outflow angle and mass flow should remain constant [2]. The corresponding formulation of the objective function is:

$$
O B F=C_{1} \cdot\left(\frac{\omega_{d}}{\omega_{\text {ref }}}\right)+C_{2} \cdot\left(\frac{\Delta \beta_{1}}{\Delta \beta_{1 . \text { ref }}}\right)+C_{3} \cdot\left(\frac{\omega_{80}}{\omega_{80 . r e f}}\right)+C_{4} \cdot\left(\frac{\sigma_{80}}{\sigma_{80 . r e f}}\right)+F
$$

In this formulation, total pressure loss at design condition is defined as $\omega_{d}$, the limits of attainable operating range, to have flat loss curve, the mean value of total pressure loss for the inner 80 percent of the operating range is called $\omega_{80}$ and corresponding standard deviation $\sigma_{80}, \mathrm{~F}$ depends on geometrical constraints.

For 3D optimization objective function is defining the mass flow, isentropic efficiency at constant mass flow with consideration of surge margin as limit. Following is the developed formulation:

$$
O B F=D_{1} \cdot\left(\frac{\text { Mass }_{d}}{\text { Mass }_{\text {ref }}}\right)+D_{2} \cdot\left(\frac{\eta_{\text {is.d }}}{\eta_{\text {is.ref }}}\right)
$$


Mass flow at 3D simulation for design condition is $\mathrm{Mass}_{\mathrm{d}}$ and isentropic efficiency at design is $\eta_{\text {is.d }}$. All of the values should be divided to the reference value, since the summation of different dimensions is permitted when all of the parameters are normalized.

$\mathrm{C}$ and $\mathrm{D}$ in both abovementioned formulations are weight factors which depend on optimizations targets.

In each blade, number of selected 2D sections depends on spanwise boundary condition variations from hub to tip and condition of three-dimensional losses, especially near the walls and locations where the effect of secondary flow is dominant. For instance, the size of near wall separation has determinative role on the selection of the near wall sections and will send them to the data base.

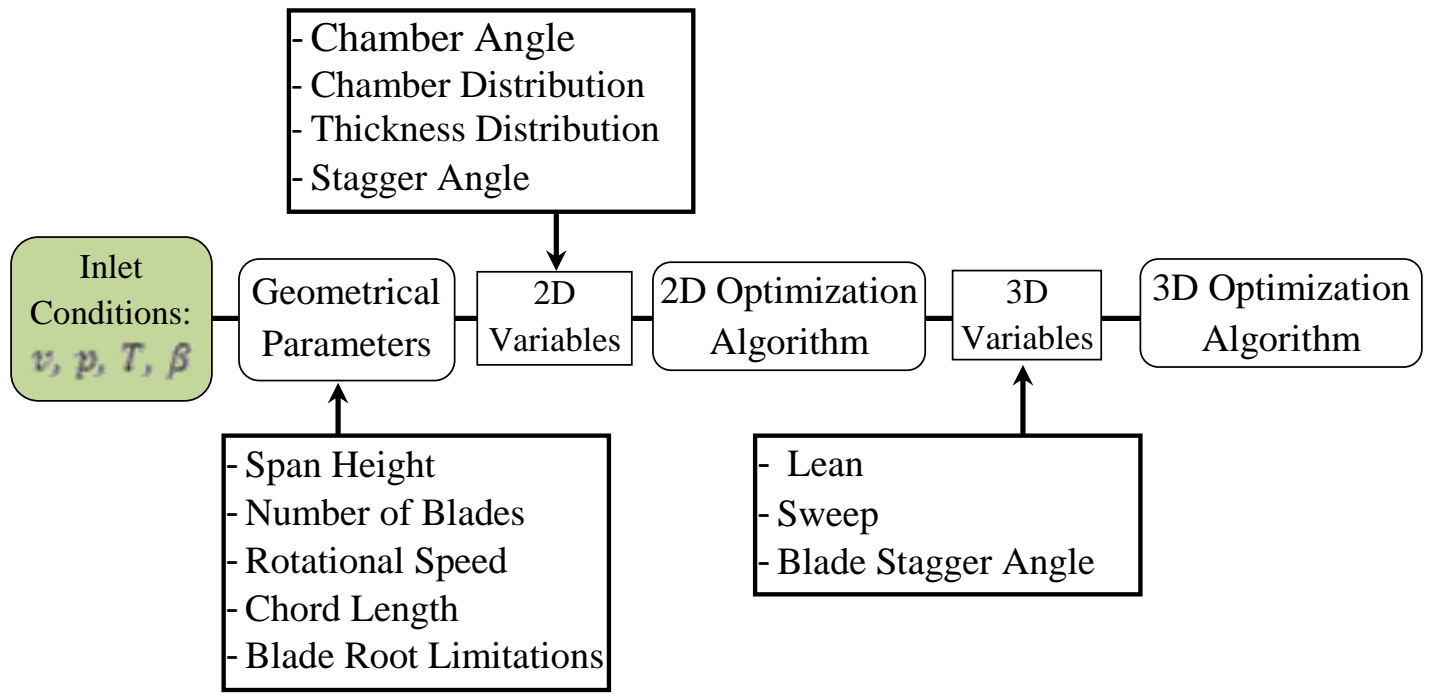

Fig. 3: Process of design blade shape.

\section{Results and Discussion}

Since the main objective of the current study is enhancement in the performance of the compressor in hot days, the optimization focused on the hot day's operation.

\subsection{Blades Optimization Procedure}

The optimization process of the airfoil was performed by the described algorithm. In addition, Genetic Algorithm (GA) tool was adjusted for 50 individuals in 50 numbers of generations.

The optimization process was performed for every eight blades (including rotary and stationary) of first four stages. Number of selected sections depends on the height of section. Considering five profiles will have a little effect on the stage total-to-total efficiency; however, it can decrease the blade surface perturbations.

Obtained results for each blade section demonstrate that the loss value was decreased to acceptable operating range for off-design operation. Afterwards, 3D simulations for total compressor stages were conducted and results compared with the primary geometry.

In order to clarify the changes made by optimization algorithm, camber line and thickness distribution for first rotor hub section has been distinct and illustrated. The optimization process starts with NACA65 profile that is defined by approximately circular arc; therefore, the curvature is constant and the position of maximum camber is at the $50 \%$ of the chord (Fig. 4). As it was indicated, the position of maximum camber line on the optimized geometry is shifted to the left side while its value compared with the base geometry is increased. Compared with the base geometry, curvature is increased in front of the optimized profile. This distribution is typically similar to CD profiles with increased curvature in front and relatively low curvature at the end of section. 
Thickness distribution curve is shown in Fig. 5 in NACA65 profiles. The maximum thickness position is located at the $40 \%$ of the chord length, while for the optimized geometry it is moved to the left and close to the leading edge. Thicker leading edge will improve the airfoil performance at off-design condition with decreased profile loss and almost constant exit flow angle.

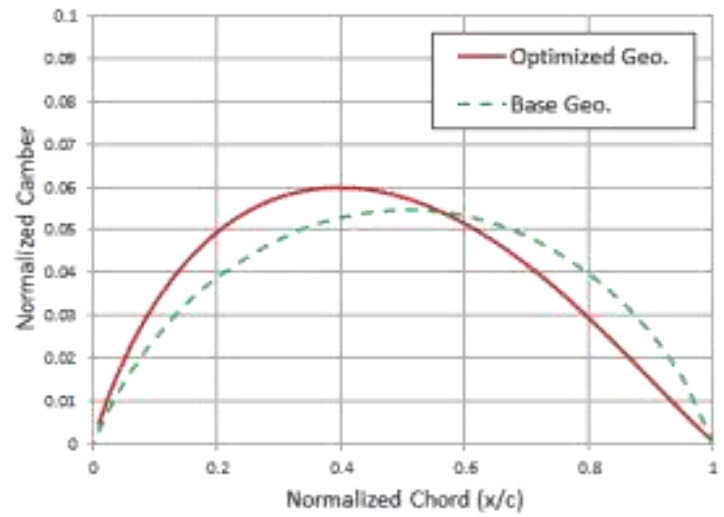

Fig. 4: Camber line distribution for the base and optimized geometries.

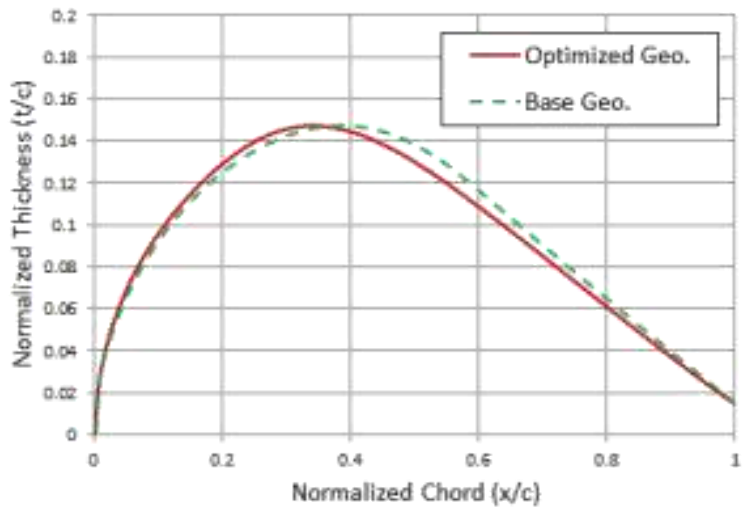

Fig. 5: Thickness distribution for the base and optimized geometries.

In the NACA65 airfoil with steeper Mach deceleration after maximum value, separation can occure sooner compared with the new profile. When the adverse pressure gradient is too strong for the flow to overcome, separation will occur (Fig. 6. $a$ and $b)$.

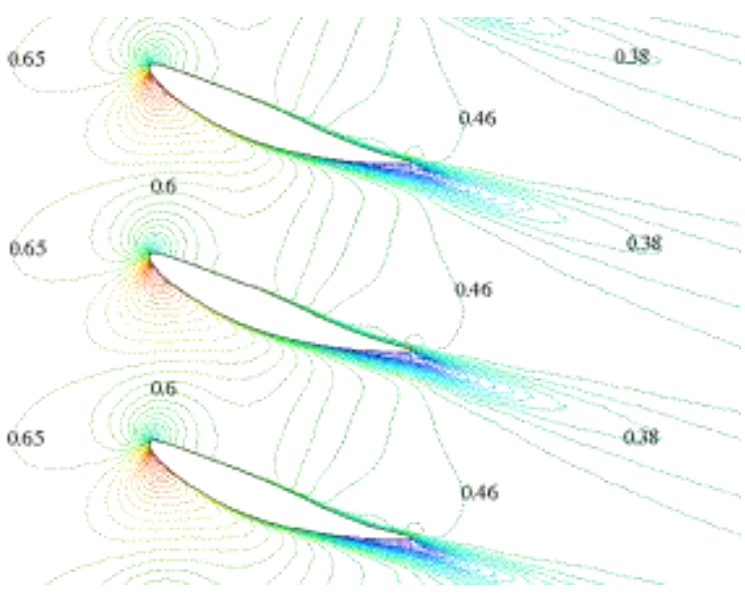

(a)

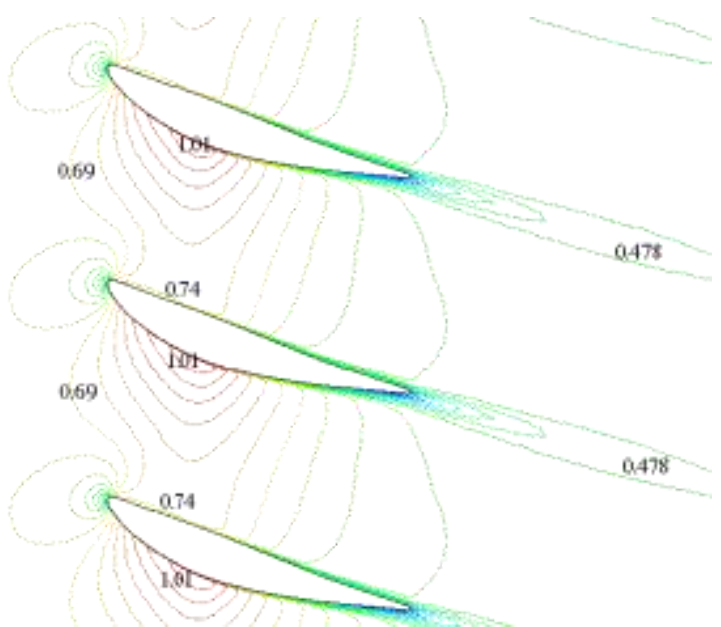

(b)

Fig. 6: Contour of much number distribution for rotor hub section at the base (a) and the optimized (b) geometries.

Fig. 7 shows the profile loss improvement for all operating condition on rotor tip section (a) hub section (b) in the second stage. It is clear that, the positive stall condition (hot days) is extended for safe operation according to its desirable off-design operating bound. 


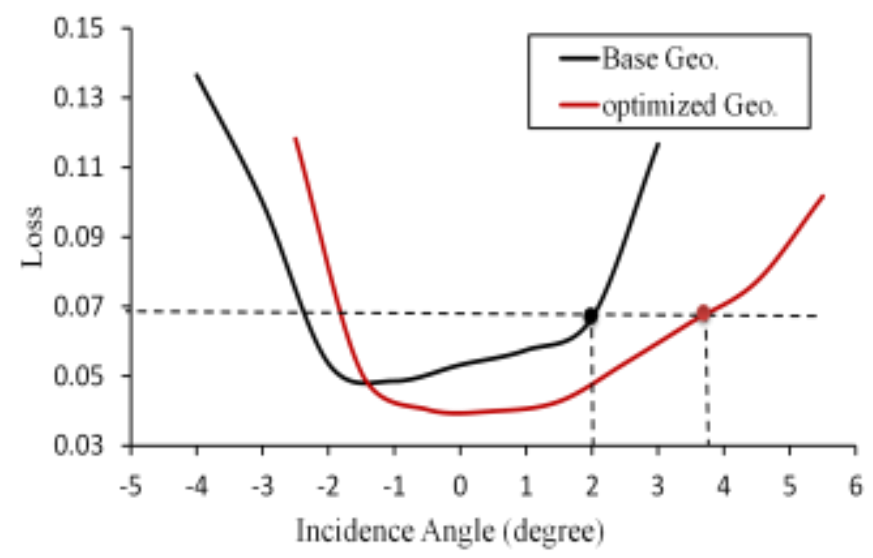

(a)

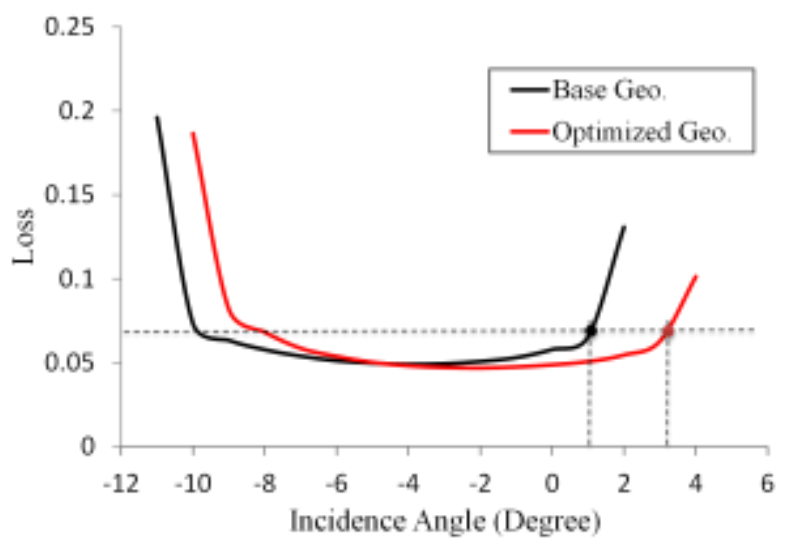

(b)

Fig. 7: loss value for starting and optimized geometry on the rotor tip section with $\mathrm{M}=0.9$ (a) rotor hub section with $\mathrm{M}=0.7$ (b).

Comparison between results of 3D simulation of base compressor and some experimental data taken from MGT-70 gas turbine during its operation in various power plants proved acceptable accuracy of the simulation. Good agreement between predictions and experimental results, both in the details of the flow field and the integral prediction of operating range of the compressor, were found. The 3D CFD simulation of compressor with replacement of first four stages with new optimized blades was employed to evaluate the results of automated airfoil design procedure. The obtained Mach number distribution for the hub section and blade of stage 2 for optimized and base geometry are compared in Fig. 8 a and b. Compared with the NACA65 blade section, the optimized profiles were more front-loaded and the position of maximum Mach number was shifted to the front side in the optimized section. It is noteworthy that minimum loss of new optimized section in the hub was decreased more than $10 \%$ and overall stage efficacy was improved up to $1.16 \%$, while total isentropic efficiency of entire 16stage compressor showed approximately $0.13 \%$ enhancement.

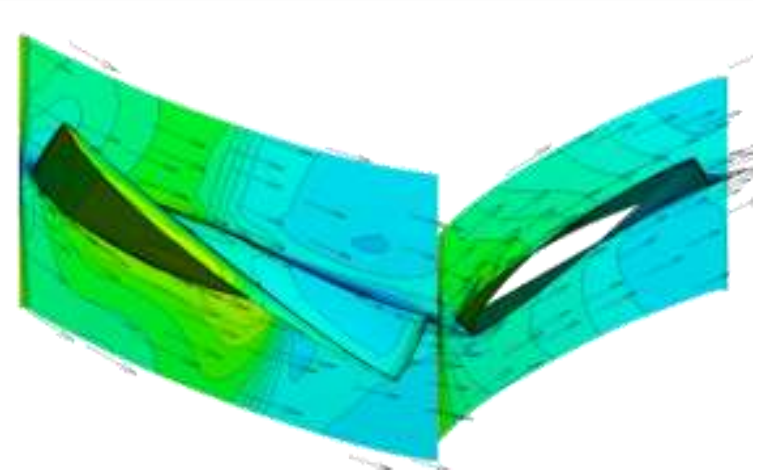

(a)

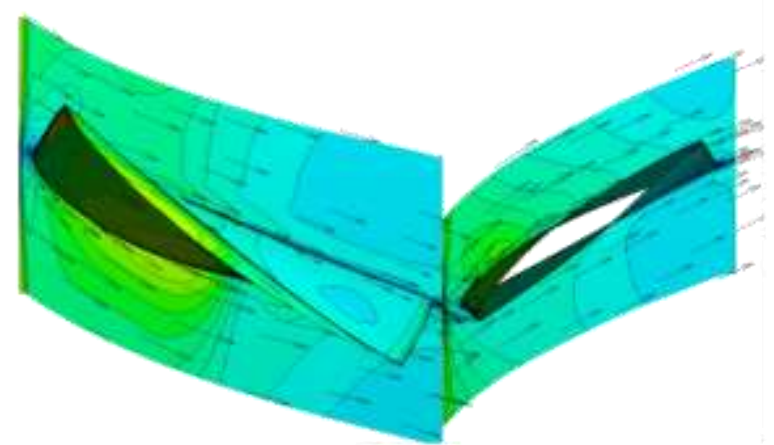

(b)

Fig. 8: Mach number distribution for generated blades with the base profiles (a) and the optimized profiles (b).

\subsection{Fully Compressor simulation with Optimized Geometry}

In order to evaluate the optimized geometry, 3D simulation was performed in design and three off-design working operation. The results of the simulations are represented in Table . 
As shown in Table, optimization of the geometry resulted in reduction in the value of inlet mass flow degradation. In addition, the inlet mass flow was enhanced by $0.85 \%$ at cold condition and continued to hot operation with $0.91 \%$. Another enhanced parameter is isentropic efficiency, about $1 \%$ and $0.91 \%$ for cold and hot conditions, respectively.

Table 3: Compressor optimized design performance (data are normalized according to starting geometry design point).

\begin{tabular}{|l|l|l|l|l|}
\hline Inlet Temperature (C) & 0 & 15 & 30 & 45 \\
\hline Corrected Speed & 1.027 & 1 & 0.975 & 0.95 \\
\hline Mass flow & 1.057 & 1.008 & 0.947 & 0.885 \\
\hline Isentropic Efficiency & 1 & 1.01 & 1.009 & 1.006 \\
\hline Pressure Ratio & 1.05 & 1.001 & 0.94 & 0.87 \\
\hline
\end{tabular}

In order to get better insight into changes in performance parameter, compressor working characteristics are shown in Fig. 9 for both the base and optimized geometries. Mass flow and efficiency improvement can be seen for each nondimensional rotational speed.

Since changes in mass flow rate can affect non-dimensional operating point of compressor, it is crucial to increase discharge pressure of compressor to achieve matched working condition with axial turbine. Since the value of increased mass flow relates with compressor exit pressure at turbine inlet condition, it is predicted that the compressor discharge pressure would be higher compared with the base geometry. As a consequence, this will effect on the surge margin in comparison with the base geometry. The surge margin of compressor is considered here based on numerical simulations. It is illustrated in this figure by black dash-line for base geometry operation and black solid line for optimized geometry.

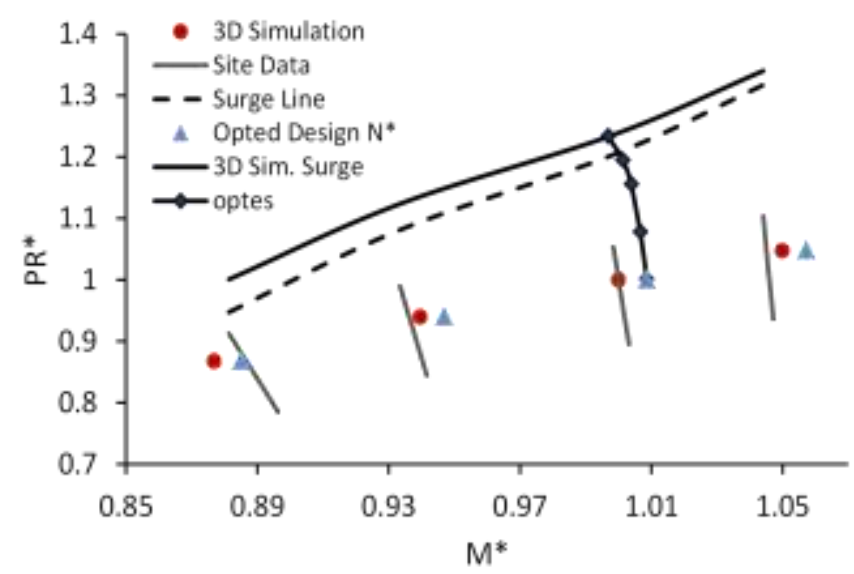

Fig. 9: Compressor characteristics for starting and optimized.

\section{Validation}

\subsection{Experimental Set-up}

Results of numerical simulation and experimental data under various conditions were compared in the current study. In order to measure flow properties, VectoFlow five-hole probes with assembled thermocouples were utilized between rotary and stator blades of the first stage (Fig. 10-a). Each five-hole probe was installed on a traverse system in order to move spanwise in the domain. Three traverse systems, located in various circumferential positions (as shown in Fig. 10-b), were applied to measure flow properties of the first stage. The outputs of the five-hole probes were connected to a pressure scanner (MIC-170) via silicon tubes. Data acquisition frequency in all experiments was equal to $10 \mathrm{~Hz}$. 

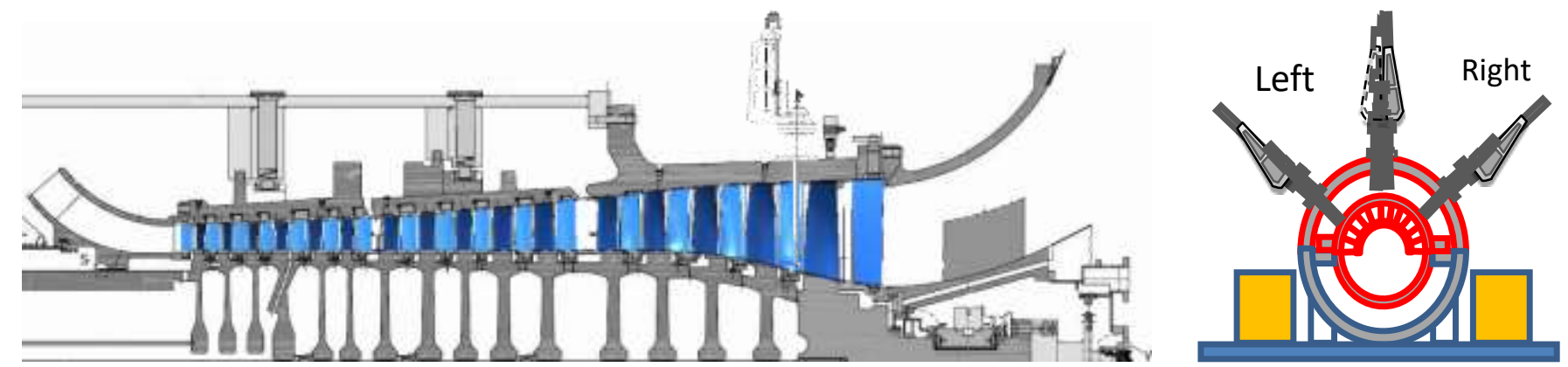

Fig. 10: Cross section of compressor with the position of centre traverse probe (a), Positions of traverse systems (b).

\subsection{Comparing numerical and experimental data}

The data obtained by traverse systems in the mentioned conditions were compared with the results of numerical simulation to evaluate the proposed model. Comparison between numerical and experimental normalized inlet flow angle is represented in Fig. 11. In this figure recorded data were normalized based on the machine base load operation in $15^{\circ} \mathrm{C}$ (triangle blue points). According to the condition of data recording of this unit, the possible recorded ambient temperature was between $5^{\circ} \mathrm{C}$ (squared red points) and $25^{\circ} \mathrm{C}$ (rounded green points). Numerical Calculated Operating Range is illustrated as blue background.

Concentration of recorded points near the walls was considered denser; this is due to higher gradient of variations in this region compared with the mid points.

Increasing inlet temperature changes the flow direction to positive range relative to $15^{\circ} \mathrm{C}$ recorded points; on the other hand, on the cool days the flow direction has negative direction.

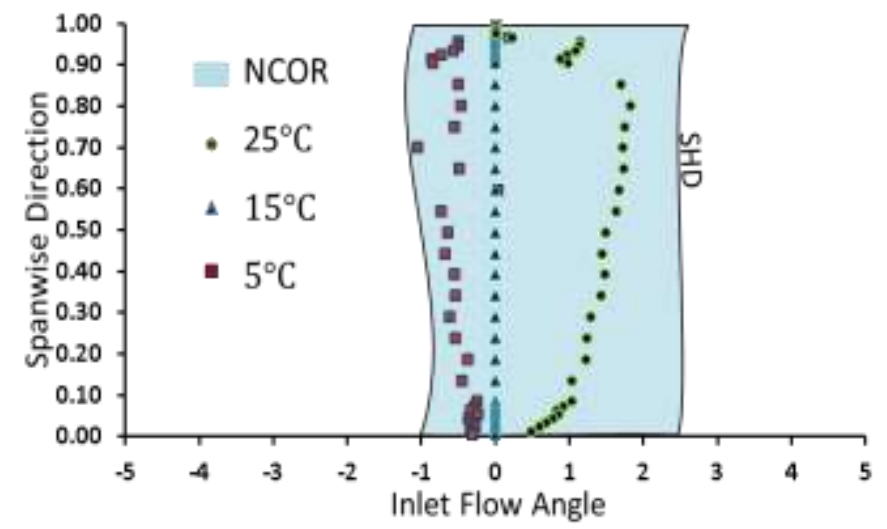

Fig. 11: Effect of ambient temperature changes from $5^{\circ}$ to $25^{\circ}$ on the first stage stator inlet flow angle.

\section{Conclusion}

In the present study, an optimization process by considering ambient temperature variations is represented. Firstly, the sensitivity of compressor to ambient temperature was investigated. Afterwards, an automated design optimization tool was developed on the basis of genetic algorithm. In this model, mechanical constraints and inlet flow angle were judged before simulation in off-design condition to decrease the computational cost.

The objective of optimization process was minimizing loss value in all working conditions by focusing on gas turbine operation in hot conditions. The geometry of the airfoil sections was changed to achieve the minimum loss value for the defined blade operation. According to the results of numerical simulation for the optimized geometries in design 
operation, the mass flow and efficiency were enhanced $0.8 \%$ and $1 \%$, respectively. Moreover, in off-design operation, at 45 degree of Celsius, $0.91 \%$ augment in efficiency was observed.

\section{Acknowledgements}

This activity is a part of Gas turbine retrofitting program in the MAPNA Turbine Engineering \& Manufacturing Co. so the writer would like to acknowledge MAPNA Company for their opportunity for publishing this report.

\section{References}

[1] H. Stephens, "Application of supercritical airfoil technology to two-dimensional compressor cascades: comparison of theoretical and experimental results," AIAA J., no. 6, pp. 594-600, 1979.

[2] U. Koller, R. Moning, B. Kusters, and H. Schreiber, "Development of advanced compressor airfoils for heavy-duty gas turbines, part I: design and optimization," ASME Paper, no. 99-GT-95, 1999.

[3] B. Kusters, H.-A. Schreiber, U. Koller, and R. Moning, "Development of advanced compressor airfoils for heavyduty Gas turbines, part II: experimental and theoretical analysis," ASME Paper, no. 99-GT-96, 1999.

[4] F. Sieverding, B. Ribi, M. Casey, M. Meyer, "Design of industrial axial compressor blade sections for optimal range and performance," ASME Paper, no. 2003-GT-38036, 2003.

[5] N. L. Sanger, "The use of optimization techniques to design-control diffusion compressor blading," ASME J. of Engineering for Power, vol. 105, pp. 256-264, 1983.

[6] M. Schnoes, and E. Nicke, "A database of optimal airfoils for axial compressor through flow design," in Proceedings of ASME Turbo Expo 2016, Seoul, South Korea, 2016.

[7] J. Alonso Reuther, A. Jameson, M. J. Rimlinger, and D. Saunders, "Constrained multipoint aerodynamic shape optimization using an adjoint formulation and parallel computers: Part ii \& I," AIAA J. of Aircraft, vol. 36, no. 1, pp. 51-71, 1999.

[8] D. Buche, G. Guidati, P. Stoll, "Automated design optimization of compressor blades for stationary large scale turbomachinery," in Proceedings of ASME Turbo Expo, USA, 2003, pp. 1-9.

[9] T. Belamri, P. Galpin, A. Braune, C. Corneljius, "CFD analysis of a 15 stage axial compressor part I: methods," ASME Turbo Expo paper, 2005.

[10] T. Belamri, P. Galpin, A. Braune, C. Cornelius, "CFD analysis of a 15 stage axial compressor part II: results," ASME Turbo Expo paper, 2005.

[11] P. Astrua P. Stefano, A. Silingardi, F. Bonzani, "Multi-Objective constrained aero-mechanical optimization of an axial compressor transonic blade," in Proc. of ASME Turbo Expo, 2012.

[12] Lars Ellbrant, Lars-Erik Eriksson, Hans Martensson, "Design of compressor blades considering efficiency and stability using CFD based optimization," in Proceedings of ASME Turbo Expo, Copenhagen, Denmark, 2012.

[13] N. Neupert, H. Gomaa, F. Joos, B. Weigand, "Investigation and modeling of two phase flow through a compressor stage : analysis of film breakup," Eur J Mech B/Fluids, vol. 61, pp. 279-88, 2016.

[14] H. S. Pham, N. Alpy, J. H. Ferrasse, O. Boutin, M. Tothill, J. Quenaut J, "Compressor: Development and qualification by means of CFD simulations," Int J Heat Fluid Flow, pp. 1-16, 2016.

[15] M. R. Pakatchian, H. Saeidi, A. Rafiei, "Axial Flow Compressor of MGT-70 Gas Turbine Blade Shape Optimization Based on Operating Condition,” IGTC2015, pp. 249-255, 2015. 